\title{
SISTEMA DE MONITORAMENTO E CONTROLE DE UM PROCESSO DE DISTRIBUIÇÃO DE ÁGUA, USANDO ARDUINO E PROTOCOLO GSM.
}

\author{
J. M. RIBEIRO ${ }^{1}$, E. C. B. F. dos SANTOS $^{1}$, G. R. OLIVEIRA ${ }^{1}$, R. A. MIRANDA ${ }^{1}$ e I. T. F. \\ RESENDE $^{1}$
}

\author{
${ }^{1}$ Instituto Federal de Ciência e Tecnologia de Sergipe \\ E-mail: eldercleiton2011@gmail.com
}

\begin{abstract}
RESUMO - O mercado atual da automação apresenta constantemente novas técnicas que podem ser utilizadas para diminuição de custos e melhoria em vários tipos de processos. Neste trabalho é apresentada uma solução eficiente, de fácil uso e baixo custo de monitoração e controle remoto de um processo real de distribuição de água desenvolvido e testado no Serviço Autônomo de Água e Esgoto - SAAE, empresa pública de saneamento, localizada no município de Estância - Sergipe, propondo a integração de um sistema de controle e automação utilizando a plataforma Arduino e o Shield SIM900. O monitoramento foi feito a partir de aparelhos celulares com padrão GSM usando comunicação SMS ou chamadas de voz. Foi desenvolvido um método que busca monitorar as temperaturas e correntes elétricas de um conjunto motor/bomba. O método aplicado no Arduino foi testado e sua eficácia foi comprovada detectando falhas provocadas por um aumento ou diminuição de temperaturas e correntes elétricas e rendimento do sistema de bombeamento de água através do envio automático de mensagens de texto para um aparelho celular cadastrado.
\end{abstract}

\section{INTRODUÇÃO}

Segundo Carrijo et al. (2003), o crescimento sem planejamento das cidades brasileiras combinado com a falta de investimentos no setor de saneamento básico tem tornado os sistemas de abastecimento de água ineficazes e de difícil operacionalidade, prejudicando a qualidade de vida da população, seja limitando o consumo essencial por parte da população, ou mesmo com a falta de qualidade devido a ausência de manutenção onde o sistema eletro-mecânico que auxilia no envio de água para a população, em alguns casos, é de difícil acesso ou carece de mão-de-obra técnica.

Dentro deste contexto a automação tem desempenhado um papel importante por possibilitar o controle e monitoramento de diversas variáveis como temperatura, nível, pressão e vazão de um determinado sistema, enviando relatórios de funcionamento, apontando exatamente as causas de um problema e possibilitando a mobilização de técnicos e de peças de forma eficiente antes mesmo de uma parada no seu funcionamento.

Dentre as tecnologias empregadas para o monitoramento e o controle de processos, o aparelho celular tem se destacado por ser uma ferramenta de fácil acesso, presente cada vez mais no cotidiano das pessoas. De acordo com trabalhos realizados por Santos et al. (2012), o uso dos 
telefones celulares está cada vez mais presente em todas as faixas etárias e classes sociais, o que faz com que o celular seja incorporado à vida cotidiana de milhões de pessoas por todo o mundo. Diante dessa realidade o número de aplicativos voltados para telefonia móvel em busca de soluções na área de automação, tem crescido significativamente pelo fato de ser uma solução eficiente, de fácil uso, baixo custo, que visa aumentar a comodidade e segurança de pessoas.

O objetivo principal deste artigo é desenvolver um sistema de controle e monitoração remoto em um conjunto motor/bombaque será aplicado de forma prática no Serviço Autônomo de água e Esgoto - SAAE, empresa pública de saneamento de água do município de Estância Sergipe, onde todas as funções possam ser gerenciadas através de mensagens de texto ou chamadas de voz pelos técnicos e pessoal cadastrado.

\section{MATERIAL E MÉTODOS}

\subsection{Arduino}

Neste trabalho o controlador utilizado foi o Arduino Mega 1280, pelo fato do mesmo ser facilmente integrado a outros dispositivos externos e possuir entradas e saídas já nomeadas que facilitam o entendimento do uso em sistemas controlados, possuindo inúmeras vantagens em relação às soluções análogas existentes no mercado.

Segundo Mcroberts (2011), dentre as vantagens do Arduino sobre outras plataformas de desenvolvimento de microcontroladores, a facilidade de utilização é a principal vantagem, por permitir que pessoas que não são da área técnica possam rapidamente aprender e criar seus próprios projetos em intervalo de tempo relativamente curto. Esse fator torna a utilização desta plataforma bastante difundida.

Outra característica importante desta plataforma em relação aos outros microcontroladores é que seus hardware e software possuem licença aberta, além de permitir a integração com vários ambientes como Linux, Macintosh e Windows, apresentando uma melhor relação de custo benefício do mercado (SANTOS, 2008). A Figura 1 mostra detalhadamente as partes e a pinagem dos soquetes do Arduino Mega 1280.

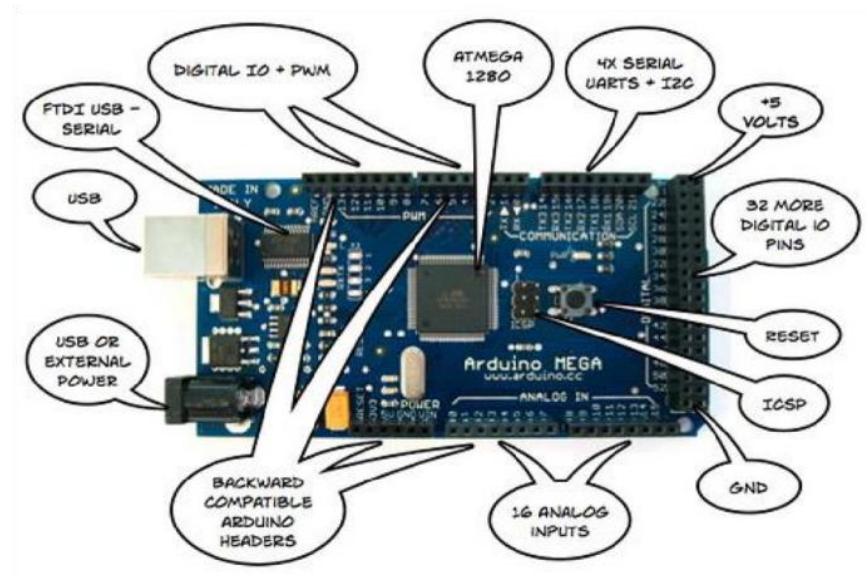

Figura 1 - Partes constituintes Arduino Mega 1280. 
Os trabalhos de SANTOS et al.(2012), mostram que esse microcontrolador possui hardware extensível permitindo encaixar em suas portas outras placas chamadas de Shields, ampliando sua funcionalidade e oferecendo suporte a outros tipos de conexão como Ethernet, Wi-fi, Bluetooth, e diversas outras.

\subsection{Modem GSM SIM900}

O Shield SIM900 é um módulo para operar na rede móvel GSM de celulares, capaz de efetuar todas as funções de um aparelho convencional como: Enviar e receber SMS, efetuar e receber chamadas e se conectar na internet. Segundo, Artur et al. (2007), os módulos GSM são soluções wireless (sem fio), que permitemo acesso a rede GSM da mesma forma que os aparelhos celulares, com a diferença está no foco do mercado está voltado para a comunicação (MáquinaMáquina), e na forma de controle destes dispositivos, feito através de Comandos AT”. A Figura 2 ilustra as principaispartes constituintes do módulo.

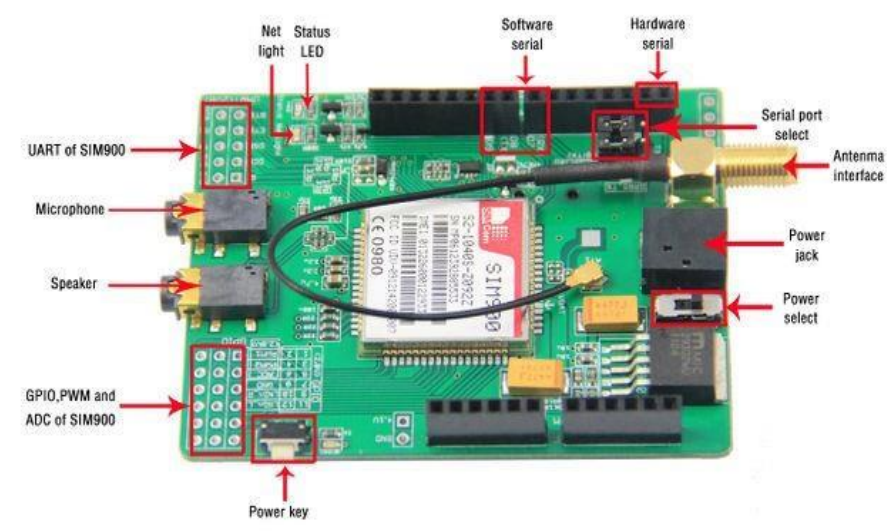

Figura 2 - Representação do Shield SIM900(modem).

Essa atual conjuntura, tem contribuído para que a maior parte das aplicações dos modens GSM esteja direcionada para transmissão de dados (SANTOS, 2012). Por conexão CSD (Circuit Switch Data), que é a conexão ponto a ponto para outro modem conectado à rede de telefonia móvel ou fixa; por conexão GPRS (General Packet Radio System), que é uma conexão na rede de pacote de dados permitindo ligação com qualquer servidor conectado à internet; e por SMS conhecido no Brasil como torpedo ou mensagem de texto, permitindo enviar ou receber 160 caracteres para um modem ou aparelho celular.

Para explorar os recursos e trabalhar com o SIM900 (modem) de maneira mais fácil, o mesmo foi conectado a um PC através de uma porta serial e a partir do uso do software emulador Hyperterminal do Windowsfoi possível testar várias funções, como por exemplo, ligar e desligar o módulo através de comandos AT no Arduino. Uma vez digitados no Hyperterminal, esse comandos foram visualizados na tela do computador. A lista com os comandos AT, suportados pelo módulo GSM é disponibilizada pelo fabricante SIMcom (MAIA, 2012). 


\subsection{Sensor de Corrente}

Os sensores de corrente são dispositivos utilizados para medir correntes de entradas numa faixa de 0 a 100A (AC), com uma saída correspondente de 0 a $50 \mathrm{~mA}$. Essa relação de transformação de corrente é realizada por transformadores de corrente (TCs) que são indispensáveis na medição de corrente alternada.

Os TCs utilizam a força do campo magnético em torno do condutor de modo a formar uma corrente induzida em seus enrolamentos secundários. Este método indireto de interface permite uma fácil instalação e proporciona um elevado grau de isolamento entre o circuito primário e circuitos de medições secundárias. A Figura 3 representa o sensor de corrente SCT-013-000 utilizado neste projeto.
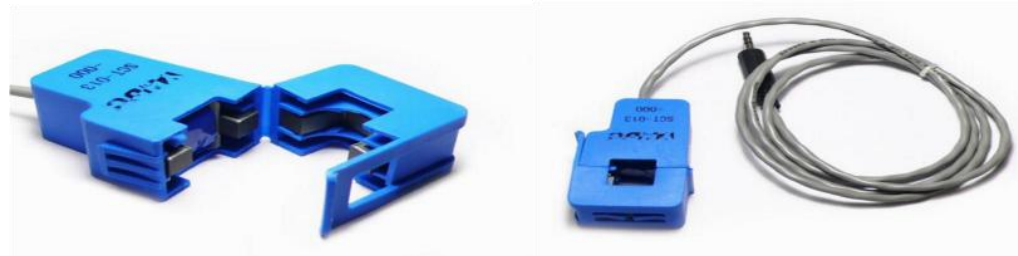

Figura 3 - Sensor de corrente SCT - 013 - 000 .

\subsection{Sensor de Temperatura}

O sensor de temperatura digital utilizado foi o DS18B20, mostrado na Figura 4, que é capaz de medir em graus Célsius e em outras escalas, além de permitir medições de temperatura com precisão em ambientes úmidos, possuindo uma resolução de 9-bit a 12-bit.

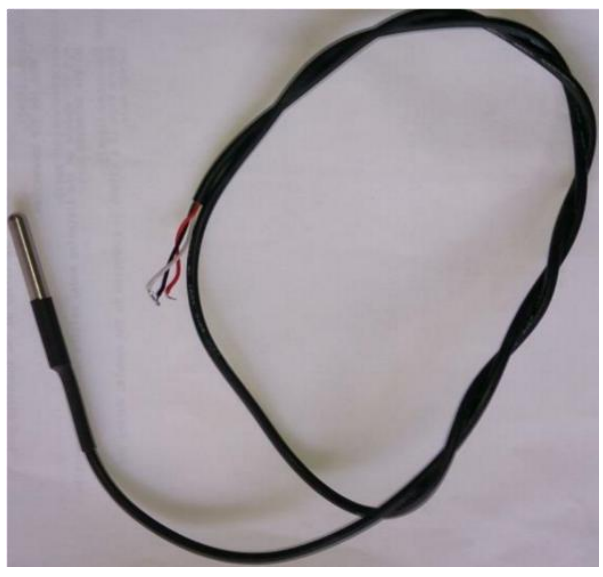

Figura 4- Sensor de temperatura DS18B20.

A comunicação é feita por 1-fio, ou seja, precisa apenas de 1 pino do microcontrolador para transferir os dados. Este dispositivo leva no máximo $750 \mathrm{~ms}$ para realizar uma conversão de 
temperatura e salvar no seu registrador correspondente, visto que neste intervalo de tempo, grandes variações de temperatura são ausentes,

Este tipo de sensor opera de $3 \mathrm{~V}$ a $5.5 \mathrm{~V}$ e pode ser alimentado pela linha de dados, podendo assim operar entre $-55^{\circ} \mathrm{C}$ até $+125^{\circ} \mathrm{C}$ e com precisão de $\pm 0.5^{\circ} \mathrm{C}$ se estiver operando dentro da faixa de $-10^{\circ} \mathrm{C}$ até $+85^{\circ} \mathrm{C}$, o qual não necessita de chips externos, visto que possuem internamente um conversor Analógico/Digital e uma memória na qual estão contidos os dados convertidos. Cada DS18B20 possui um número serial único de 64-bit, o que permite que vários sensores funcionem no mesmo barramento.

\subsection{Metodologia}

Inicialmente foi feita a seleção e o estudo das bibliotecas GSM-Shield (modem), DallasTemperature (sensor de temperatura) e EmonLib (sensor de corrente) que foram adotadas durante o desenvolvimento do projeto. Uma vez escolhidas as bibliotecas o próximo passo foi estudar cada componente de forma minuciosa tanto na parte de hardware como no software, a fim de verificar se atenderia o objetivo do projeto, realizar o controle e a monitoração remota de temperatura e corrente de um conjunto motor/bomba, visando sempre o baixo custo.

Após a escolha de cada componente, iniciou-se o estudo detalhado do Shield SIM900 (modem) através do Monitor Serial do Arduino que permitiu explorar os diversos comandos AT'snecessários para realizar chamadas e enviar SMS's.

Em seguida o outro componente estudado foi o sensor de temperatura DS18B20 que possui função de leitura, conversão de escalar de temperatura, dentre outras funções. Com os sensores ligados ao Arduino foi possível através da biblioteca (Dallas-Temperature) obter os endereços físicos de cada sensor. Neste trabalho foram utilizados apenas dois sensores, com a finalidade de monitorar a temperatura do motor e da bomba centrifuga, respectivamente.

Por último, foi estudado o sensor analógico de corrente SCT-013-000 que possibilitou a leitura de corrente elétrica alternada, neste projeto foram utilizados três sensores, a fim de medir as correntes elétricas de um motor trifásico de indução (MIT).

A etapa final da parte física consistiu na montagem de todas as partes em um recipiente de fácil fixação nos trilhos internos do Centro de Controle de motores (CCM). A Figura 5 mostra como ficou o protótipo completo ligado aos sensores de corrente e temperatura. Foi feita a montagem deste protótipo na captação de água bruta Biriba II, responsável por abastecer um populoso bairro chamado Cidade Nova. 

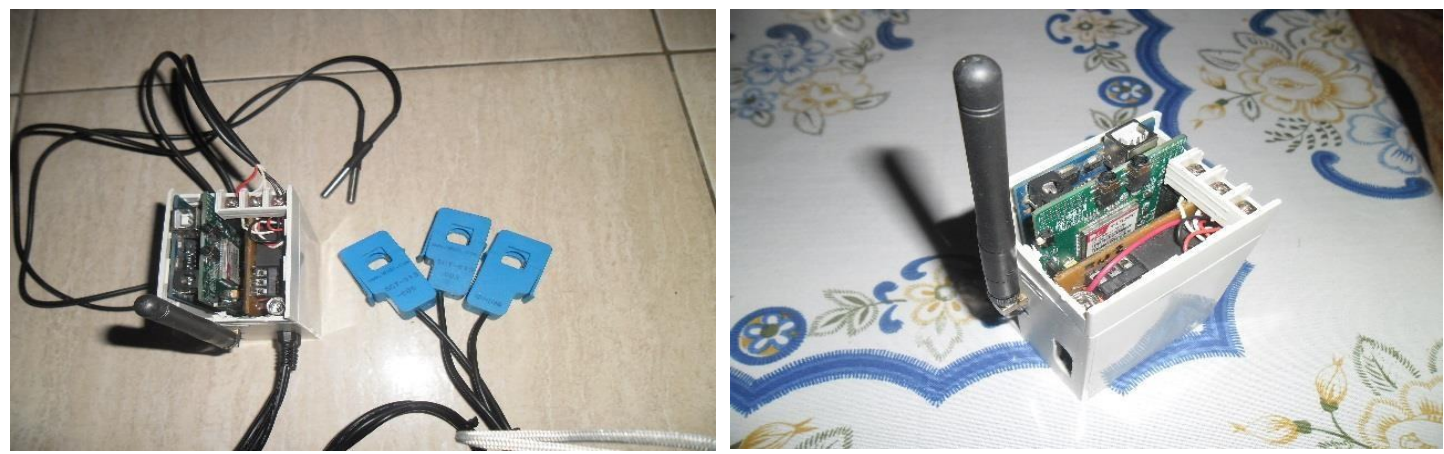

Figura 5 - Protótipo completo com Sensores de temperatura e corrente.

O conjunto motor/bomba do sistema em estudo possui uma potência nominal de $75 \mathrm{CV}$ e uma tensão de alimentação de 380 V com uma corrente nominal de 100A em carga plena. Porém, nesse sistema o MIT trabalha ocioso com corrente de 75 A em cada uma das três fases. Na Figura 6 é mostrado o CCM e o conjunto motor/bomba, no interior do CCM foi instalado o protótipo.
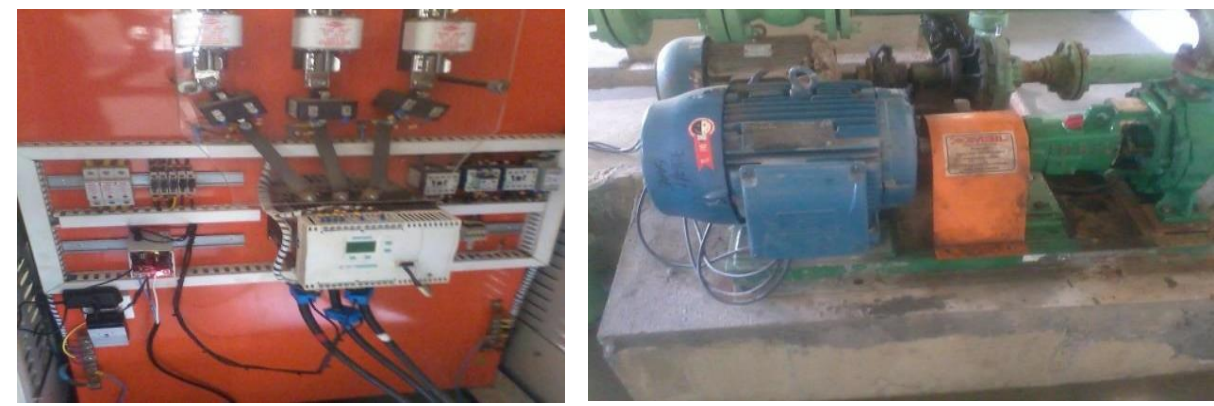

Figura 6 - Centro de comando de motores e conjunto motor/bomba.

\section{RESULTADOS E DISCUSSÃO}

O sistema proposto proporcionou a partir de uma simples mensagem de texto ou uma chamada de voz de usuários cadastros, o controle e monitoramento do conjunto motor/bomba, com base no envio de um SMS ou uma ligação para o SIM900 (modem). Neste momento uma série de condicionais são verificadas pelo Arduino, como por exemplo, se a mensagem de texto ou o número pressionado durante uma chamada são compatíveis com a Tabela 1 abaixo para que seja efetuada uma determinada ação.

Tabela 1 - Mensagens de texto e tons DTMF's aceitos e suas funcionalidades

\begin{tabular}{|l|c|c|}
\hline MENSAGEM & CHAMADA & FUNCIONALIDADE \\
\hline Bomba liga & DTMF: 1 & Liga sistema \\
\hline Bomba desliga & DTMF: 2 & Desliga sistema \\
\hline Temperatura & DTMF: 3 & Retorna temperaturas \\
\hline Corrente & DTMF: 4 & Retorna correntes \\
\hline Status & DTMF: \# & Retorna relatório completo \\
\hline
\end{tabular}


Na Figura 7 é demonstrado o funcionamento do modem GSM ao receber uma chamada, se o número estiver cadastrado a ligação é completada e tão logo o usuário pode discar as teclas compatíveis com a Tabela 1. Para receber um relatório completo do estado do conjunto motor/bomba é necessário pressionar a tecla "\#” (sustenido) ou enviar um SMS com a instrução "Status". Após este procedimento é retornado ao usuário, por mensagem de texto, a ação solicitadacomo verificar se o sistema está ligado ou desligado ou os valores das correntes elétricas e das temperaturas.
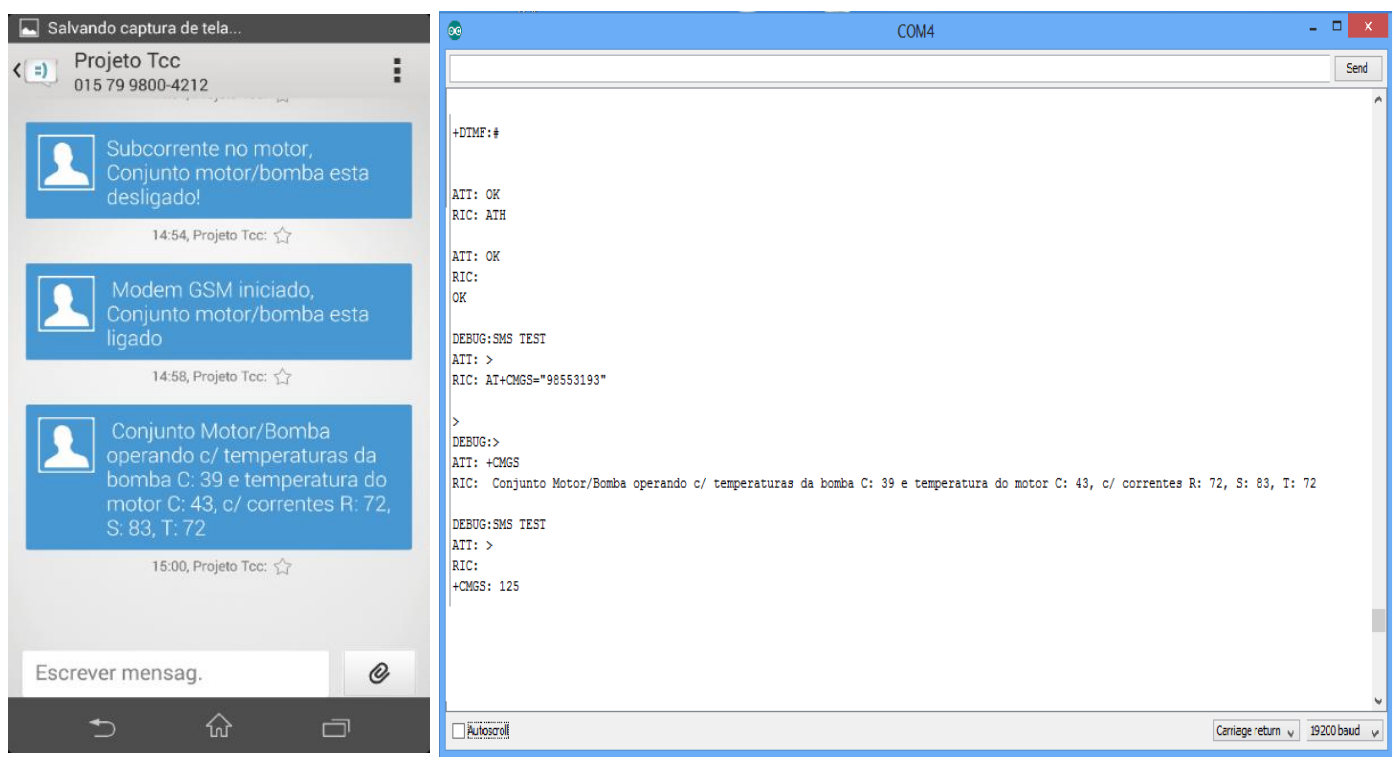

Figura 7 - Comunicação entre celular e SIM900 mostrada pelo Serial Monitor.

Enquanto aguarda o recebimento de SMS's ou chamadas, um constante monitoramento dos valores de corrente elétrica e temperatura é realizado. De acordo com os resultados obtidos na Figura 8, observa-se que os valores lidos pelos sensores foram de 39 e $43^{\circ} \mathrm{C}$, respectivamente, para a temperatura da bomba centrífuga e do MIT. Caso a temperatura ultrapasse $60^{\circ} \mathrm{C}$ em quaisquer dos sensores o conjunto motor/bomba é desligado imediatamente e um SMS é retornado para todos os usuários cadastrados.

Para efeitos de comparação foi instalada no painel do CCM uma Interface Homem-Máquina - IHM, onde foram mostrados os valores das correntes elétricas das três fases por um display LCD. Os resultados mostraram que os valores das correntes elétricas monitoradas pelos sensores de corrente registraram, aproximadamente os mesmos valores da IHM: fase 1: 71A, fase 2: 82A, fase 3: 71A. 


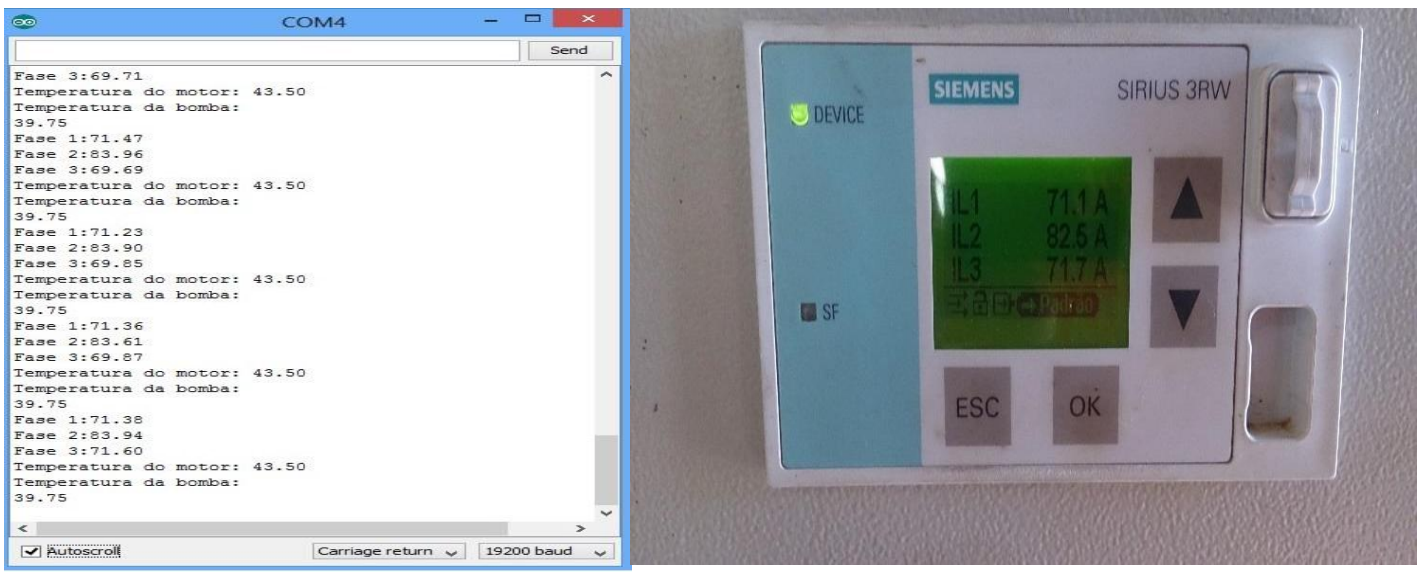

Figura 8 - Monitoramento das correntes elétricas e temperatura pelo Serial monitor e pela IHM.

\section{CONCLUSÕES}

De acordo com os resultados obtidos, observou-se que o sistema proposto se comportou conforme o esperado, sendo capaz de fornecer com precisão os principais parâmetros do sistema estudado, como corrente e temperatura. A proposta de se utilizar o protocolo GSM se mostrou como uma alternativa viável por permitir além do monitoramento do sistema, a intervenção em caso de alguma anormalidade, o que torna o sistema desenvolvido como sendo uma ferramenta promissora em sistemas de abastecimento situados em áreas remotas. Desta forma, podemos concluir que o sistema de monitoramento e controle desenvolvido pode ser utilizado como uma ferramenta importante para garantir a otimização de processos industriais. A otimização também é importante no tratamento da água, visto que, os recursos e produtos utilizados para o tratamento da mesma podem ser utilizados de forma mais eficiente.

\section{REFERÊNCIAS}

ARTUR, R; FIGUEIREDO, R; NASCIMENTO, L.H.B. Projeto de um controlador de alarme de carro via SMS. Universidade Estadual de Campinas - SP, 2007.

CARRIJO, I. B.; REIS, L. F. R.; CHEUNG, P. B.; SOARES, A. K.;SILVA, F. G. B. Otimização da operação de sistemas de distribuição de água utilizando algoritmos genéticos multiobjetivo, disponível em: http://www.bwd.com.br/geasd/fotos/gea_publicacoes18.pdf. Acesso em: 18 de janeiro de2011.

FREDRIZZI, M. C. Fornecimento de água com sistemas de bombeamento fotovoltaico. São Paulo, 1997.

MAIA, G. M. F. Acionamento remoto de portões elétricos via celular através de microcontrolador. Centro UniversitáriodeBrasília (UniCEUB), 2012.

MCROBERTS, M. Arduino Básico.Novatec Editora. São Paulo, 2011

SANTOS, N.P. Introdução ao Arduino. 17 ed. rev. Portugal; Programar, 2008.

SANTOS, E. C.B.F.; RIBEIRO, J.M.; GIACOMELLI, W. Automação residencial: sistemas microcontrolados com comunicação wireless via GSM. CONNEPI: Palmas 2012. Disponível em: <http://propi.ifto.edu.br/ocs/index.php/connepi/vii/paper/view/1043>.Acesso em: [20 fev 2014]. 